\section{IMPROVING CPGS IMPLEMENTATION BY INTEGRATIVE PROGRAMS: EXAMPLE OF THE ELECTIVE CAESAREAN SECTION}

S Musset, M Martinowsky, A Danscoine, R Bataillon, M Laurence. Haute Autorité de Santé, Paris, France

\section{0:1136/bmjqs-2013-002293.102}

Background In France, one in five women gives birth by caesarean section and in almost half of cases, caesarean section is planned. Elective caesarean is a subject of controversy (some indications, maternal request) and its practice is marked by highly disparities in the country.

Objective To improve the relevant practice of elective caesarean. Methods In a short time, an integrative process was developed with systematic reviews, working group of multidisciplinary health professionals and patients, focus group, implementation network.

Results For serving the identified needs, we updated guidelines, developed information for/with the patients, and produced implementation tools adapted to the different medical indications. Clinical situations which may lead to a planned caesarean section are varied and complex. For each of them, we redefined the indications of caesarean section or vaginal delivery. For elective caesarean, women should be informed as soon as possible, with provided developed tools, to enable an informed discussion with the caregiver and to improve the maternal experience. The decision elements to report in the medical record were defined and a set of tools with the approach 'patient journey' developed: clinical pathways, evaluation criteria of relevance or grids, examples of improvement actions and indicators. In February 2013, more than 183 establishment of health were engaged in the programme.

Conclusions We provided a set of implementation tools to improve the relevance of planned caesarean section. The continuous feedback by the different actors will allow enriching electronic version tools and sharing knowledge in the field, thus enabling a continuous improvement process.

\section{VALIDATION OF THE GUIDELINE IMPLEMENTABILITY FOR DECISION EXCELLENCE MODEL (GUIDE-M)}

${ }^{1} \mathrm{M}$ Brouwers, ${ }^{2} \mathrm{M}$ Kastner, ${ }^{1} \mathrm{~J}$ Makarski, ${ }^{1} \mathrm{~L}$ Durocher. ${ }^{1}$ Department of Oncology, McMaster University, Hamilton, Canada; ' Li Ka Shing Knowledge Institute of St. Michael's Hospital, Toronto, Canada

\section{0:1136/bmjqs-2013-002293.103}

Background We developed a Guideline Implementability for Decision Excellence-Framework Model (GuIDE-M) based on the robust evidentiary base of a realist review on guideline attributes. GuIDE-M emerged as a conceptual representation of factors to facilitate the development of more implementable guidelines.

Objectives Validity assessment of GuIDE-M with international guideline developers.

Methods We assessed GuIDE-M using a stepwise validation process: Stage 1 involved consultation with a multi-disciplinary group of Canadian experts (including guideline research, psychology, management, and human factors engineering) to assess the sense and structure of the conceptual GuIDE-M. In Stage 2, 200 international guideline developers will assess GuIDE-M using an innovative online assessment platform, which includes an interactive video-based system to enable objective assessment of the model and its components.
Results In Stage 1, consultation with 10 multi-disciplinary experts informed major structural changes (e.g., addition of a 6th domain) and minor sense changes (e.g., collapsing like attributes) to the model. Stage 2 (in progress) will assess the organisation of the model according to Stage 1 findings: 1) To consider Stakeholder involvement, Evidence synthesis, Considered judgement and Feasibility in the development of guidelines; 2) and to communicate this content using effective Language and Format. Discussion We are applying an innovative stepwise process to rigorously validate and refine GuIDE-M internationally.

Implications for Guideline Developers/Users This study represents a novel contribution to guideline developers and will offer a comprehensive, validated model that considers an exhaustive set of evidence-based factors to facilitate guideline uptake.

\section{IMPLEMENTING UPDATED US FEDERAL GOVERNMENT RECOMMENDATIONS ABOUT HIV PREVENTION FOR PERSONS WITH HIV: A HIGH-IMPACT APPROACH FOR HIV PREVENTION}

${ }^{1} \mathrm{~K}$ Irwin, ${ }^{2} \mathrm{~A}$ Huang, ${ }^{1} \mathrm{P}$ Wortley, ${ }^{1} \mathrm{~A}$ Patel, ${ }^{1} \mathrm{G}$ Dumitru. ${ }^{1}$ US Centers for Disease Control and Prevention, Atlanta, USA; ${ }^{2}$ Health Resources and Services Administration, Rockville, USA

\section{0:1136/bmjqs-2013-002293.104}

Background More than one million people are living with HIV in the US; each year this number increases by $\sim 30,000$. Nearly two-thirds of new infections occur in racial/ethnic minorities. The 2010 National HIV/AIDS Strategy emphasises cost-effective, high-impact strategies that reduce the infectiousness of HIVinfected persons over behavioural interventions for HIV-uninfected persons. To advance this Strategy, the US Department of Health and Human Services consolidated and updated existing federal government recommendations for HIV prevention for persons with HIV.

Objectives Describe guideline development and implementation methods.

Methods HIV prevention, care, and policy experts from CDC, HRSA, NIH and six non-governmental organisations updated existing federal government recommendations for clinicians, non-clinical prevention providers, health departments, and policy makers based on new evidence about biomedical, behavioural, and policy interventions.

Results The updated recommendations emphasise new, and effective, but under-used interventions: evidence-based linkageto-care strategies, early initiation of antiretroviral therapy, interventions supporting adherence to suppress viral load and reduce infectiousness, and new behavioural interventions. To implement guidelines, CDC and HRSA are coaching "champions" in public and private sectors; disseminating decision-support tools and low-cost implementation resources, aligning federal policies with the guidelines; advising states about regulations that promote implementation; and evaluating guideline adherence and enablers through national studies.

Discussion Implementing these broad, inter-sector recommendations will require long-term engagement of governmental and non-governmental organisations. Successful implementation promises to invigorate America's HIV prevention strategy, help reduce health disparities, and decrease the nation's devastating HIV burden.

Implications for Guideline Developers/Users Understanding the benefits of consolidated guidelines that advance national policy. 